\title{
Design of a Linearized Magnetic Spring for Body-Worn Inertial Energy Harvesters
}

\author{
Hui Huang, Geoff V. Merrett, Neil M. White \\ Electronics and Computer Science \\ University of Southampton, SO17 1BJ, UK \\ $\{$ hh2g09, gvm,nmw $\} @$ ecs.soton.ac.uk
}

\begin{abstract}
A potential method for powering body-worn sensors is that of inertial energy harvesting; extracting energy from the movement of the human body. However, the frequencies typically present are $<5 \mathrm{~Hz}$, hence requiring physically large devices. A promising solution utilizes a magnetic spring, but these exhibit a non-linear relationship between force (and hence resonant frequency) and displacement. This paper describes a design for implementing a linearized magnetic spring. Finite element analysis is used to model this device and compare against those reported in the literature. Simulation results indicate that, compared to the state-of-the-art, this design exhibits improved linearity $(2 \%)$ across a wider displacement range $( \pm 25 \mathrm{~mm})$. A prototype has been fabricated, and the simulation results experimentally validated.
\end{abstract}

\section{INTRODUCTION}

Applications of body-worn sensors, which are continuing to increase in popularity while decreasing in physical size, usually require inconvenient or cumbersome battery replacement or recharging [1]. Ideally, prolonged or even unlimited operation without intervention is desired from such devices. A promising way of addressing this is to make use of inertial energy harvesting, which converts the movement of the human body into electrical energy to power the device [2-4]. Inertial energy harvesters are often designed as resonant spring-mass systems, whereby maximum power output is obtained when the frequency of movement equals the resonant frequency of the harvester. Previous studies have shown that the frequencies present on the body during activities such as walking and running are less than $5 \mathrm{~Hz}$ [5], and our own research has identified a requirement for a body-worn harvester that has a resonant frequency of $3.9 \mathrm{~Hz}$ [6]. This is the motivation for the work presented in this paper.

One of the simplest designs for an inertial energy harvester is a cantilever, with one end of the beam fixed and the other free to move (usually supporting an added mass). The resonant frequency of the system can be reduced by increasing the length of the beam and increasing the mass. However, in the case of body-worn devices, physical dimensions and weight are both parameters that need to be minimized in order to gain user-acceptance. Some attempts have been made to reduce the physical dimensions of a cantilever-based system by shaping the beam, for example by coiling it into a spiral [7]. However, even in these cases, the relatively low stiffness of the beam coupled with the end mass causes considerable bend in the beam at its quiescent position; this will increase the necessary size of the generator in an additional dimension, further increasing the volume. Other techniques have been proposed for implementing low-frequency generators, for example those using frequency up-conversion [8] or non-resonant operation [9], but these are outside the scope of this paper.

To constrain the volume of a low-frequency inertial generator, a design utilizing a mechanical spring in a tube can be used. However, there are many difficulties in fabricating a spring to produce a resonant frequency of $3.9 \mathrm{~Hz}$. Therefore the cost and durability of such devices is prohibitive. Magnetic springs, which exploit the repulsive force between two poles, are a potential alternative. These offer advantages over mechanical springs including simpler design, easier tuning of the resonant frequency, and lower mechanical fatigue [10].

$$
k=\frac{F}{x}
$$

Mechanical springs have a constant stiffness, $k$, which means that the resultant force $F$ is proportional to the displacement $x$ from its quiescent position (1). Unlike mechanical springs, magnetic springs have a nonlinear forcedisplacement relationship, and hence a variable stiffness. As a result, and as shown by (2), the resonant frequency $f_{0}$ of the magnetic spring varies with displacement (where $m$ is the mass of the moving object) [11]. The resonant generator desired by our application is required to operate rotated at different angles with respect to gravity. As a result, the quiescent position of the moving mass will change, and hence the resonant frequency needs to remain constant across the full range of displacement.

$$
f_{0}=\frac{1}{2 \pi} \sqrt{\frac{k}{m}}
$$

In this paper, we propose a novel design for a linearized magnetic spring for use in low-frequency energy harvesting applications. Our design is compared to existing devices that have been reported in the literature $[10,12]$ through Finite Element Analysis (FEA) modeling and simulation. The proposed design exhibits a spring stiffness of $134 \mathrm{~N} / \mathrm{m}$ with a linearity of under $2 \%$ over a displacement range of -25 to 25 $\mathrm{mm}$. The maximum displacement of $25 \mathrm{~mm}$ can be achieved if the magnetic spring vibrates with an acceleration of $15 \mathrm{~m} / \mathrm{s}^{2}$ at a frequency of $3.9 \mathrm{~Hz}$. This reflects an improvement over the state-of-the-art, and the design is practically validated through the fabrication and analysis of a prototype device. 

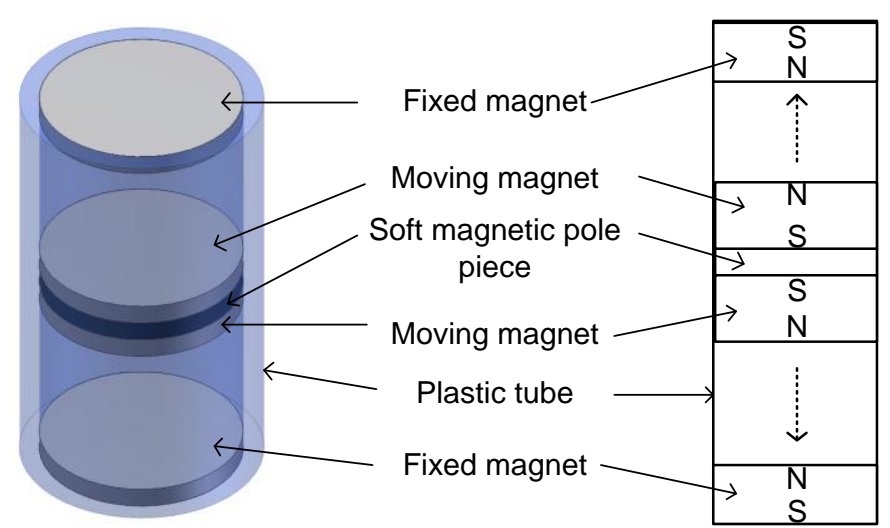

Figure 1. Structure of the magnetic spring reported by Saha et al. [10]

\section{ANALYSIS OF EXISTING WORK}

This section describes the design of a magnetic spring previously reported as providing linear-operation for a bodyworn energy harvester with a low resonant frequency [10]. The device, which had a resonant frequency of $8 \mathrm{~Hz}$, was designed to harvest energy during walking and slow running. The magnetic spring (shown in Fig. 1) was formed from two identical cylindrical magnets (diameter: $10 \mathrm{~mm}$, length: $1 \mathrm{~mm}$ ) fixed at both ends of a Teflon tube. Suspended between these were two cylindrical magnets (diameter: $15 \mathrm{~mm}$, length $8 \mathrm{~mm}$ ) separated by a soft magnetic pole piece (diameter: $15 \mathrm{~mm}$, length $3 \mathrm{~mm}$ ), glued together so that they moved as a single object. The complex design of this moveable mass was to increase the flux density to produce an increased output voltage. The separation between the middle object and either end magnet was $16 \mathrm{~mm}$, and the length of the tube $55 \mathrm{~mm}$. The authors report that the relationship between the force and the displacement of the central mass from the center is considered "almost linear" over the displacement range of -8 to $8 \mathrm{~mm}$ from the quiescent position.

Using the dimensions reported by Saha et al., we modeled and analyzed their device using FEA and compared the results with those shown in the paper (see Fig. 2). In Fig. 2, the solid line shows the simulation results of displacement against the force applied to the magnetic spring, and shows good correlation with the results reported in the paper (shown by the crosses). The independent linearity of the simulation results (the dashed line in Fig. 2, with a spring stiffness of $45.8 \mathrm{~N} / \mathrm{m}$ ) is $6.3 \%$. As discussed in the introduction, a constant spring stiffness is desired for a body-worn energy harvester, providing a linear force-displacement relationship.

In order to meet our requirement for an inertial generator with a resonant frequency of $3.9 \mathrm{~Hz}$, the quiescent position of the central magnet, when mounted vertically, is $16 \mathrm{~mm}$ from the center of the device due to gravity [5]. Thus, the region over which the spring behaves linearly will be greater than 16 $\mathrm{mm}$. The device proposed by Saha et al. was adapted in simulation to give it a resonant frequency of $3.9 \mathrm{~Hz}$, increasing the separation between end magnets to $33 \mathrm{~mm}$. This achieves a displacement of $25 \mathrm{~mm}$ with a force of $0.4 \mathrm{~N}$, considering a moving central mass of $27 \mathrm{~g}$.

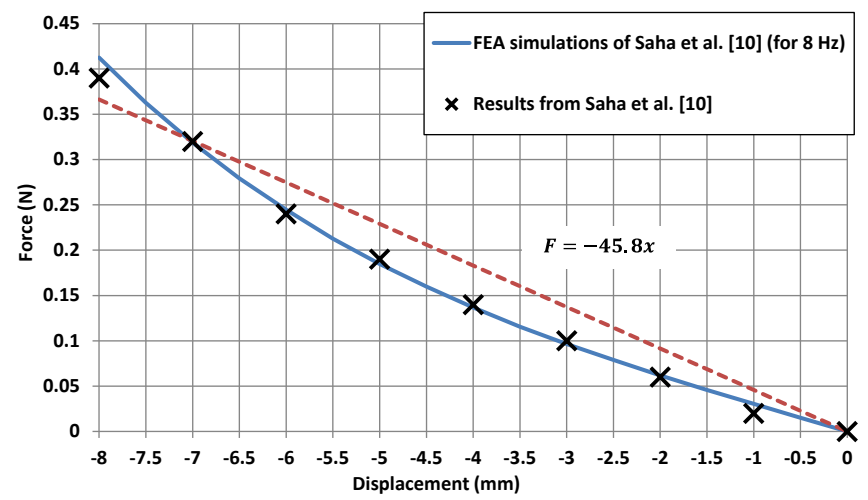

Figure 2. Force vs. displacement for the magnetic spring reported by Saha $e$ al. [10]. The solid line shows results of FEA simulation, the crosses are results from the original paper, and the dashed line shows desired linear operation.

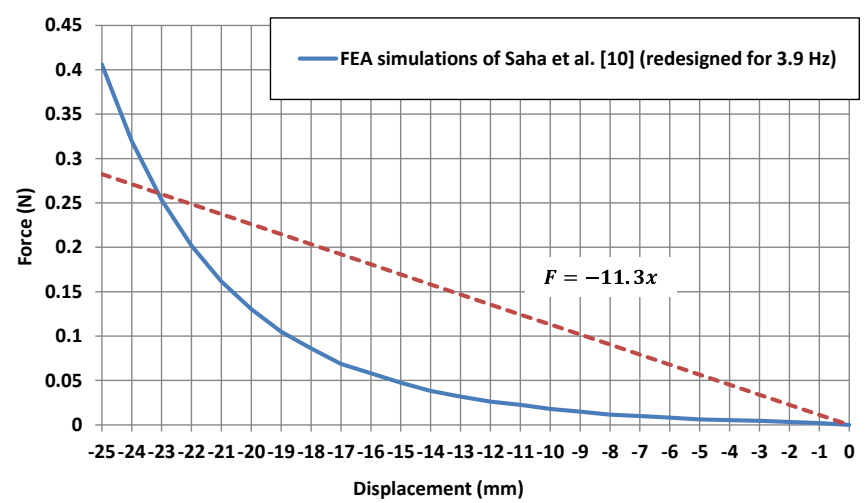

Figure 3. Force vs. displacement for the structure proposed by Saha et al. [10], redesigned for $3.9 \mathrm{~Hz}$. The solid line shows results of FEA simulation, and the dashed line shows desired linear operation.

However, as can be seen from the simulation results (Fig. 3 ), the independent linearity of the redesigned $3.9 \mathrm{~Hz}$ magnetic spring is $21.9 \%$; clearly more linear operation is desired. For our low-frequency energy harvester, we require a magnetic spring with a linearity of $<5 \%$ over a displacement range of $>16 \mathrm{~mm}$. In order to linearize the operation, different magnet arrangements were investigated which would allow 'shaping' of the magnetic field. One such solution was based upon that reported by Patt [12], the structure of which is shown in Fig. 4. The spring is formed from two radially poled ring magnets fixed at both ends of a tube. Suspended between these is a single object formed from two magnetic rings. A structure based around this design (many parameters were not reported in the paper) was modeled and analyzed using FEA simulation, and the results are shown in Fig 5. While this device is much stiffer (requiring $>50 \mathrm{~N}$ to displace the central magnet by 3 $\mathrm{mm}$ ), it is a promising solution to linearize operation.

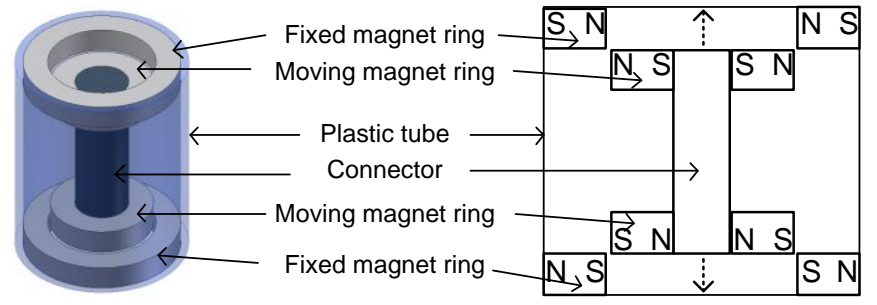

Figure 4. Structure of the magnetic spring reported by Patt [12]. 


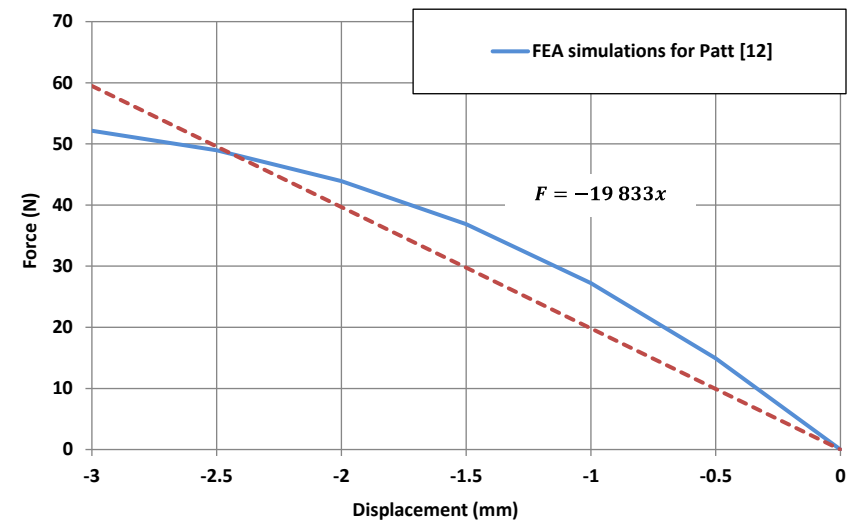

Figure 5. Force vs displacement of the magnetic spring reported by Patt [12] The solid line shows results of simulation, the dashed line linear operation.

\section{PROPOSED LINEARIZED MAGNETIC SPRING}

A design which merges the concepts reported by Saha et al. [10] and Patt [12] was designed, which utilizes a cylindrical center magnet and ring-shaped end magnets (as shown in Fig. 6). The double center magnet in Saha et al.'s design was not adopted, as the increased flux density that this would have offered necessitates a longer tube to operate at $3.9 \mathrm{~Hz}$. Axially poled magnet rings were used instead of radially poled ring magnets to significantly reduce the tooling cost. A permanent magnet was placed inside a low-friction plastic tube, and two ring magnets fixed at either end. The center magnet is free to move but suspended due to the repulsive forces.

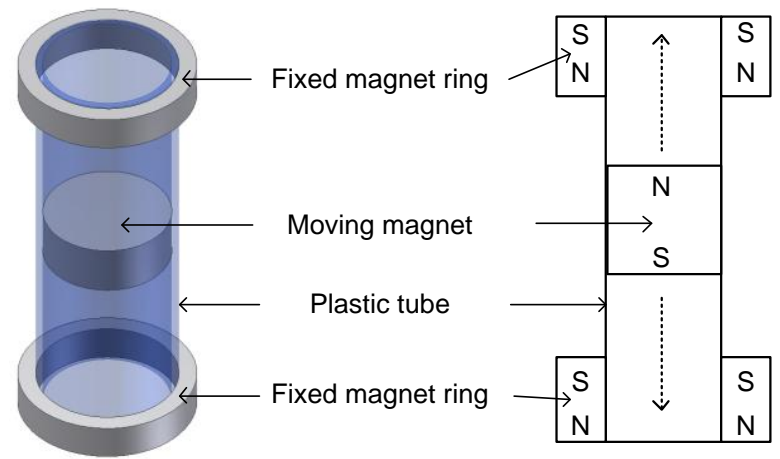

Figure 6. Structure of the magnetic spring proposed in this paper.

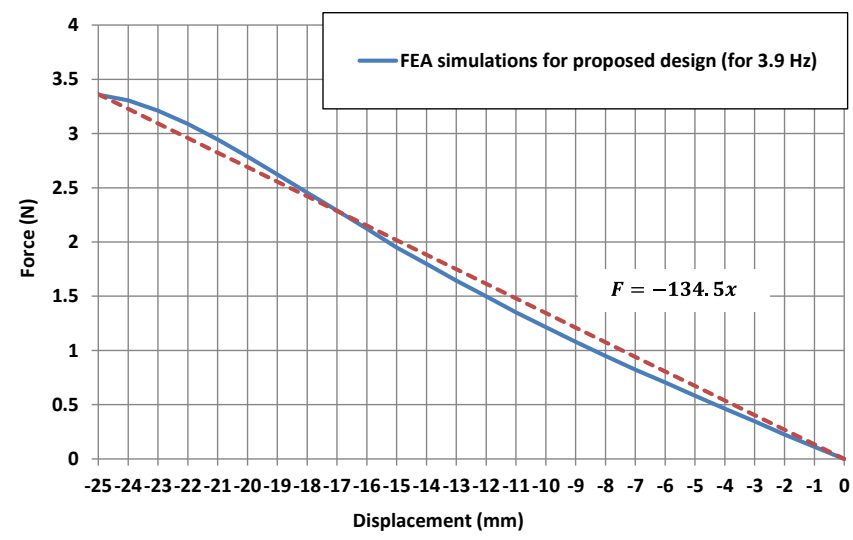

Figure 7. Force vs. displacement for the proposed magnetic spring (designed for $3.9 \mathrm{~Hz}$ operation). The solid line shows results of FEA simulation, and the dashed line shows desired linear operation.
The linearized magnetic spring was designed and optimized using FEA and the force-displacement relationship of the central magnet investigated. In order to achieve a resonant frequency of $3.9 \mathrm{~Hz}$ and ensure a linear performance, the separation between the magnets, the dimensions and the materials were carefully chosen. The solid line in Fig. 7 shows how the displacement of the central mass varies with force between -25 and $0 \mathrm{~mm}$. The independent linearity of the simulation results (the dashed line in Fig. 7, with a spring stiffness of $134 \mathrm{~N} / \mathrm{m}$ ) is $1.9 \%$. This represents a significant improvement over previously reported work.

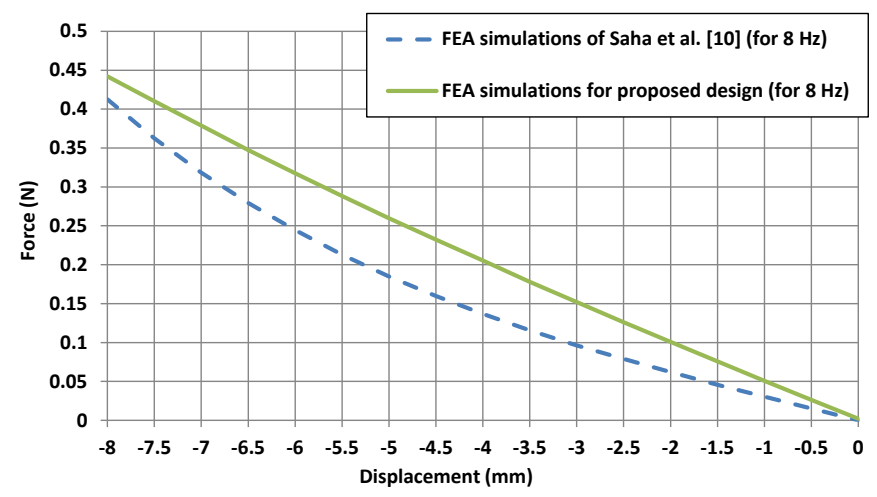

Figure 8. Force vs. displacement for the magnetic spring (dashed line) reported by Saha et al. [10] and our proposed $8 \mathrm{~Hz}$ structure (solid line)

For comparison with the device proposed by Saha et al., our proposed device (Fig. 6) was modified to tune the spring stiffness to a suitable level for a resonant frequency of $8 \mathrm{~Hz}$. The fixed magnet rings (inner diameter: $15 \mathrm{~mm}$, outer diameter: $23 \mathrm{~mm}$, length: $3 \mathrm{~mm}$ ) and central magnet (diameter: $14 \mathrm{~mm}$, length $4 \mathrm{~mm}$ ) were chosen. The total length of the device is $56 \mathrm{~mm}$ which is close to the length of the device reported in the literature. The dashed and solid lines in Fig. 8 show simulation results based on the reported and the proposed designs respectively. The linearity of our proposed structure is $1.2 \%$ compared to that reported in the literature $(6.3 \%)$.

\section{EXPERIMENTAL VALIDATION}

Two prototypes of the linearized magnetic spring were fabricated, and the force vs. displacement relationship was tested. Permanent magnets made of NdFeB N38M were inserted into the hollow plastic tubes made of Tufset polyurethane (chosen to minimize friction), while two permanent magnet rings made of $\mathrm{NdFeB} \mathrm{N} 42$ were glued to both ends of each tube with opposing polarity. The separation between the middle and either end magnet was $40 \mathrm{~mm}$. The holders for the end magnets were made from Tufset in one version of the prototype, and Delrin in the other. The complete specification of the magnetic springs is given in Table 1, and Fig. 10 shows a photo of one prototype besides an AA battery.

In order to evaluate the linearity of the fabricated device, the displacement of the central magnet with applied force was tested. The device was secured to a surface, and a wire glued to the central magnet. This wire was connected to a digital force meter (SPC Technology; SLD 5FGN) with a range of $\pm 50 \mathrm{~N}$ and resolution of $10 \mathrm{mN}$ [13], and pulled in line with the axis of the device over a range of 0-25 $\mathrm{mm}$ (in $1 \mathrm{~mm}$ increments). 
TABLE I. DETAILS OF THE PARTS USED IN THE PROTOTYPE DEVICES

\begin{tabular}{|c|c|c|c|}
\hline Part & Dimensions (mm) & Weight (g) & Material \\
\hline $\begin{array}{l}\text { Outer magnet } \\
\text { rings }\end{array}$ & $\begin{array}{l}\text { Outer diameter: } 40 \\
\text { Inner diameter: } 23 \\
\text { Height: } 6\end{array}$ & 37.6 & NdFeB N38M \\
\hline $\begin{array}{l}\text { Central } \\
\text { magnet }\end{array}$ & $\begin{array}{c}\text { Diameter: } 22 \\
\text { Height: } 8\end{array}$ & 22.8 & NdFeB N42 \\
\hline \multirow{2}{*}{ Tubes } & \multirow{2}{*}{$\begin{array}{c}\text { Outer diameter: } 24.4 \\
\text { Inner diameter: } 22.4 \\
\text { Height: } 88\end{array}$} & $\begin{array}{l}27.5 \text { (Delrin } \\
\text { holders) }\end{array}$ & \multirow{2}{*}{$\begin{array}{c}\text { Tufset } \\
\text { Polyurethane }\end{array}$} \\
\hline & & $\begin{array}{c}24.3 \text { (Tufset } \\
\text { holders) }\end{array}$ & \\
\hline
\end{tabular}

Measurements were repeated five times for each of the two prototypes; the results are shown in Fig. 9, which shows the range of measurements observed at each displacement. The results from the first prototype (Tufset end holders) exhibit a linearity of $2.0 \%$, while the second prototype (Delrin end holders) exhibits a linearity of $1.7 \%$. Comparing these results with the simulation results shows good correlation, and highlights the ability of our magnetic spring to operate with good linearity over a displacement of -25 to $25 \mathrm{~mm}$. It can be observed from Fig. 9 that there is some discrepancy between the experimental results and simulations. This difference is due to a number of factors, including measurement error (a ruler was used to measure displacement), and difficulty in aligning the magnets exactly parallel with each other. Furthermore, even with lubricant used inside the tubes, friction between the moving magnet and the tube affects the required force.

The spring stiffness for the two prototypes are $128 \mathrm{~N} / \mathrm{m}$ and $145 \mathrm{~N} / \mathrm{m}$ at the quiescent position. Hence, by adding masses of $190 \mathrm{~g}$ and $218 \mathrm{~g}$ to the middle magnets respectively, a resonant frequency of $3.9 \mathrm{~Hz}$ is obtained for both prototype devices. While the contribution of this paper is not in proposing an energy harvester, the power from a device using our spring would be comparable to that achieved by Saha et al. $(2.5 \mathrm{~mW})$.

\section{CONCLUSIONS}

This paper presents a linearized magnetic spring for the low-frequency operation of body-worn inertial energy harvesters. FEA simulations show that the proposed design has a linearity of $1.9 \%$ over a displacement range of $50 \mathrm{~mm}$, representing a significant improvement over reported devices.

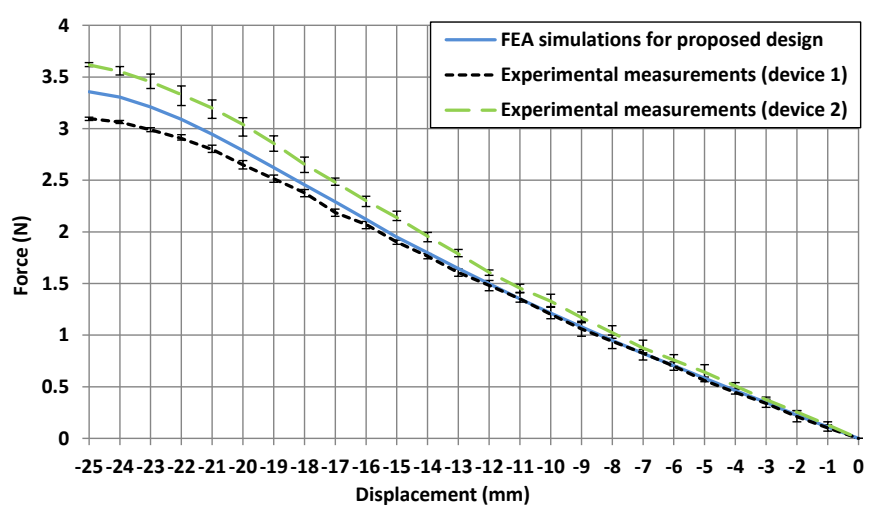

Figure 9. Measurements of force vs. displacement from the two prototype devices. Simulation results are shown for comparison.

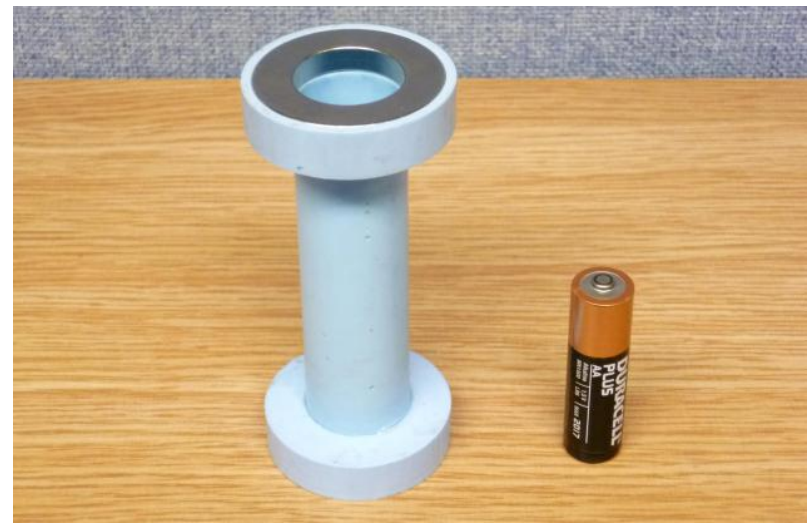

Figure 10. Photograph of the developed prototype alongside an AA battery.

A prototype has been fabricated to validate the design, and the force vs. displacement relationship measured. The results showed good correlation with the FEA simulations. Our ongoing research is in utilizing the developed linearized magnetic spring to design and fabricate an electromagnetic body-worn inertial energy harvester.

\section{REFERENCES}

[1] G. Z. Yang, Body sensor networks: Springer, ISBN 1-84628-272-1. 2006, pp. 1-40.

[2] T. V. Buren, P. D. Mitcheson, T. C. Green, E. M. Yeatman, A. S. Holmes, and G. Troster, "Optimization of inertial micropower generators for human walking motion," IEEE Sensors Journal, vol. 6 , pp. 28-38, 2006.

[3] V. Bedekar, J. Oliver, and S. Priya, "Pen harvester for powering a pulse rate sensor," Physics D-Applied Physics, vol. 42, p. 105105 (9pp), 2009.

[4] L. C. Rome, L. Flynn, E. M. Goldman, and T. D. Yoo, "Generating electricity while walking with loads," Science, vol. 309, pp. 1725-1728, 2005.

[5] T. V. Buren, "Body-worn inertial electromagnetic micro-generators," Ph.D. dissertation, Swiss federal institute of technology Zurich, ETH Zurich, 2006.

[6] H. Huang, G. Merrett and N. White, "Human-powered inertial energy harvesters: the effect of orientation, location and activity on obtainable power," in Eurosensors XXV, 4-7 September 2011, Athens, Greece, 2011.

[7] W. J. Choi, Y. Jeon, J. H. Jeong, R. Sood, and S. G. Kim, "Energy harvesting MEMS device based on thin film piezoelectric cantilevers," Journal of Electroceramics, vol. 17, pp. 543-548, 2006.

[8] I. Sari, T. Balkan, and H. Kulah, "An electromagnetic micro power generator for low-frequency environmental vibrations based on the frequency upconversion technique," Journal of Microelectromechanical Systems, vol. 19, pp. 14-27, 2010.

[9] M. Renaud, P. Fiorini, R. Schaijk, and C. Hoof, "Harvesting energy from the motion of human limbs: the design and analysis of an impactbased piezoelectric generator," Smart Materials and Structures, p. 035001 (16 pp.), 2009.

[10] C. R. Saha, T. O'Donnell, N. Wang, and P. McCloskey, "Electromagnetic generator for harvesting energy from human motion," Sensors and Actuators A: Physical, vol. 147, pp. 248-253, 2008.

[11] S. Beeby, M. Tudor, and N. White, "Energy harvesting vibration sources for microsystems applications," Measurement Science and Technology, vol. 17, pp. R175-R195, 2006.

[12] P. Patt, "Design and testing of a coaxial linear magnetic spring with integral linear motor," Magnetics, IEEE Transactions on, vol. 21, pp. 1759-1761, 1985

[13] SLD 5FGN digital force gauge datasheet [Online]. Available: http://www.newark.com/pdfs/datasheets/spc/Ta-971.pdf 